\title{
Colitis, independent of macronutrient intake, compromises bone structure and strength in growing piglets
}

\author{
Photios Vassilyadi', Scott V. Harding ${ }^{1,2}$, Tom J. Hazell ${ }^{1,3}$, Hope A. Weiler ${ }^{1}$ and Linda J. Wykes ${ }^{1}$
}

BACKGROUND: Deterioration in bone health is a concern in managing pediatric inflammatory bowel diseases, but clear understanding of the independent contributions of disease and nutrition is lacking. This study aimed to ascertain whether bone health could be conserved during colitis by maintaining adequate nutritional intake in growing piglets.

METHODS: The effect of colitis on bone structure and strength was determined in piglets with dextran sulphate sodium-induced colitis. Piglets received either 100\% macro/ micronutrient requirements or 50\% macro/100\% micronutrient requirements. Femurs were analyzed for dual-energy $x$-ray absorptiometry, peripheral quantitative computed tomography, microcomputed tomography, and 3-point bending tests. RESULTS: Colitis, regardless of a well-nourished or malnourished diet, compromised areal bone mineral density (-17\%) and volumetric bone mineral density $(-20 \%)$ in cortical and trabecular bone. Structural integrity at mid-diaphysis was maintained during colitis; however, lower cortical area, trabecular area, and bone mineral content resulted in lower energy to break.

CONCLUSION: Colitis compromises both bone structure and strength of long bones in piglets, independent of macronutrient intakes. Although confirmation of these findings in pediatric cohorts is needed, these data identify aspects of bone health that may be affected by inflammatory bowel disease.

n

nflammatory bowel disease (IBD) in children causes slowed growth, impaired bone growth, and decreased stature (1-4). Children with IBD present with faltering linear growth and are often shorter (mean z-score -0.5) than expected for age compared with their parents and peers with up to $12.2 \%$ of this group being classified as stunted ( $<-2 \mathrm{z}$-score) (5). Similarly, dual-energy $\mathrm{x}$-ray absorptiometry (DXA) imaging reveals that $75-89 \%$ have low areal bone mineral density (aBMD) during active disease states and $10-37 \%$ havelow aBMD $(\mathrm{z}$-score $<-2.5)$ when in remission (6).

Long bones (e.g., femur and tibia) of children and adults are affected differently by IBD as pediatric populations usually present with slowed growth, while adults show signs of increased bone resorption (7). Abnormal bone development and skeletal morbidity may be multifactorial (8) or associated with inflammation (9), side-effects of medications (10) and/ or inadequate nutrition (11). It has also been suggested that decreased physical activity and muscle strength may affect bone health in pediatric IBD (12).

Malnutrition is common among children with IBD and may be the result of reduced intake (13). Early studies demonstrated that bones of severely malnourished children are shorter and lighter than healthy children (14), and children with edematous malnutrition showed growth restriction, irregularly calcified epiphyses, and diminished pattern of trabeculae in both femur and tibia (15). If bone resorption is elevated and bone formation is reduced, bone modeling would be altered and reflected in bone geometry-ultimately, this would negatively affect bone strength. However, to date, such data in pediatric IBD is scarce (7).

Macronutrient under-nutrition, especially protein malnutrition, has been studied extensively in rats. Growing rats fed a protein-restricted diet (2.5\% casein) for $16 \mathrm{wk}$ had decreased aBMD, bone mineral content (BMC), and bone strength in the lumbar spine, femur, and tibia, along with lower plasma insulin-like growth factor I (IGF-I) levels compared with rats fed a protein adequate diet (15\% casein) (16). In a similar study, femurs of growing rats fed a $4 \%$ protein diet had lower linear dimension, weight and strength than controls receiving a $20 \%$ protein diet (17).

The dextran sulphate sodium (DSS) model of colitis is a wellestablished animal model of inflammation and mucosal injury similar to that of human ulcerative colitis $(18,19)$. Our group has developed a piglet model of colitis (20-23) because the gastrointestinal physiology, morphology, and pathology of the piglet more closely resemble that of humans (24). In this study, we investigated how the structure and strength of femurs were affected by colitis and nutrition in growing piglets. Specifically, we used DXA, peripheral quantitative computed tomography (pQCT) and micro-computed tomography $(\mu \mathrm{CT})$ in our analysis of long bone (whole, trabecular, and cortical) with respect

\footnotetext{
'School of Dietetics and Human Nutrition, McGill University, Montreal, Quebec, Canada; ${ }^{2}$ Diabetes and Nutritional Sciences Division, Faculty of Life Sciences and Medicine, King's College, London, UK; ${ }^{3}$ Department of Kinesiology and Physical Education, Wilfrid Laurier University, Waterloo, Ontario, Canada. Correspondence: Linda J. Wykes (linda.wykes@mcgill.ca)

Received 13 April 2016; accepted 2 May 2016; advance online publication 3 August 2016. doi:10.1038/pr.2016.135
} 


\section{Articles | Vassilyadi et al.}

to bone area, $\mathrm{BMC}$, aBMD, volumetric bone mineral density (vBMD) and cortical thickness. We also used the 3-point flexure method to determine bone strength.

The aim of this study was to ascertain whether bone structure and strength could be maintained during colitis by providing adequate macronutrient intake. Specifically, we hypothesized that: (i) malnourished piglets with colitis (MN-C)would have decreased bone area, BMD, BMC, cortical thickness, and bone strength compared with healthy, well-nourished piglets, and that (ii) well-nourished piglets with colitis would maintain bone health similar to wellnourished piglets without colitis.

\section{RESULTS}

As previously described by Harding and colleagues, colitis was confirmed by the presence of fecal occult blood by day 9 of the 14-day study and colon histology was characterized using a 6-point scale with 4 sub-classifications (higher histological damage scores were demonstrated in piglets with colitis than health controls) (21). Well-nourished piglets with colitis (WN-C) demonstrated lower increases from Day 1 in chest circumference $(-33 \%)$ and snout-to-rump length $(-22 \%)$ compared with healthy piglets without colitis (WN-Healthy). Malnourished piglets with colitis demonstrated lower gains in average daily weight $(-63 \%)$ and lower increases from Day 1 in chest circumference $(-77 \%)$ and snout-to-rump length $(-29 \%)$ compared with healthy piglets (22). In this study, results are presented in graphical form, while data tables can be found in supplemental materials. In addition, an analytical model of the left femur was created to clearly portray our methodology and gain a visual appreciation of the level of detail captured by DXA, pQCT, and $\mu \mathrm{CT}$ (Figure 1).

\section{Linear Dimensions}

Femur length was not different among the three groups $(7.11 \pm 0.14 \mathrm{~cm})$. Neither widths $(1.14 \pm 0.03 \mathrm{~cm})$ nor cortical thicknesses $(0.10 \pm 0.01 \mathrm{~cm})$ at mid-diaphysis were different among groups (see Supplementary Table S1 online).

\section{Whole Bone Analysis}

Femur aBMD was affected by both colitis and macronutrient intake (Figure 2). The DXA analysis confirmed lower whole bone aBMD of femur in WN-Colitis $\left(0.242 \mathrm{~g} / \mathrm{cm}^{2}\right)$ by $16 \%(P<0.05)$ and MN-Colitis $\left(0.239 \mathrm{~g} / \mathrm{cm}^{2}\right)$ by $17 \%(P<0.05)$ compared with WN-Healthy $\left(0.287 \mathrm{~g} / \mathrm{cm}^{2}\right)$. Whole bone area and $\mathrm{BMC}$ were not different among groups, whereas $\mu \mathrm{CT}$ analysis demonstrated no difference in whole bone volume, BMC, and vBMD among groups (see Supplementary Table S2 online).

\section{Bone Geometry Analyzed by pQCT}

The pQCT analysis of femurs $4 \%$ from the growth plate revealed lower vBMD (total, trabecular, and cortical) in WN-Colitis (189, 173 , and $219 \mathrm{mg} / \mathrm{cm}^{3}$ respectively) by $20 \%(P<0.05)$ compared with WN-Healthy $(243,230$, and $276 \mathrm{mg} / \mathrm{cm}^{3}$ respectively); however, area (total, trabecular, and cortical) was not different (Figure 3). Similar patterns of

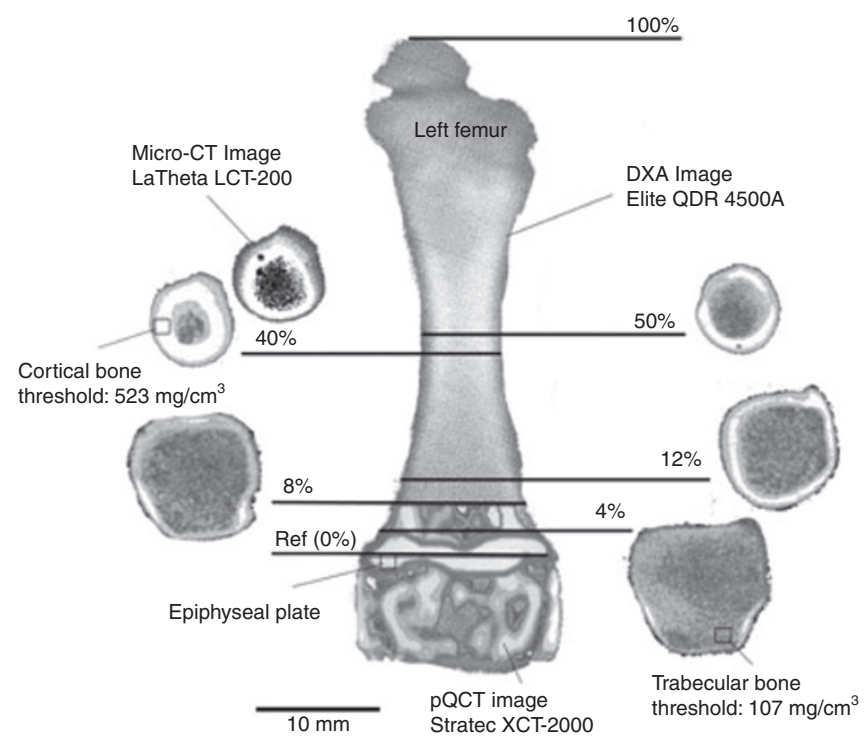

Figure 1. Analytical model of left femur - anterior view, proximal (femoral head) to distal. Dual-energy x-ray absorptiometry (DXA) image of the whole bone, appreciating the 2-dimentional, areal view of the femur. Peripheral quantitative computed tomography (pQCT) image of the distal femur is superimposed onto the DXA image, appreciating the 3-dimentional, volumetric view of the femur. The epiphyseal plate sets the $0 \%$ reference point. Select slices measuring trabecular bone are shown 4,8 , and $12 \%$ from the epiphyseal plate, while slices measuring cortical bone are shown 40 and $50 \%$ from the epiphyseal plate. Micro computed tomography $(\mu \mathrm{CT})$ image at mid-diaphysis (corresponding to the $\mathrm{PQCT}$ site $40 \%$ from the epiphyseal plate), appreciating the similarity between $\mu C T$ and $\mathrm{pQCT}$.

change were seen 8 and $12 \%$ from the growth plate. At the $40 \%$ site, lower cortical area in WN-Colitis $\left(27 \mathrm{~mm}^{2}\right)$ by $21 \%$ $(P<0.05)$ and $\mathrm{MN}$-Colitis $\left(27 \mathrm{~mm}^{2}\right)$ by $21 \%(P<0.05)$ was observed compared with WN-Healthy $\left(34 \mathrm{~mm}^{2}\right)$; however, cortical vBMD was not different. Neither cortical area nor vBMD were different $50 \%$ from the growth plate (see Supplementary Table S3 online).

\section{Bone Geometry at Mid-Diaphysis by $\mu \mathrm{CT}$}

Femur area at mid-diaphysis was uniquely affected by colitis and nutritional status (Figure 4). Cortical and trabecular area of femur at mid-diaphysis were lower in $\mathrm{WN}$-Colitis $(0.28$ and $0.10 \mathrm{~cm}^{2}$ respectively) by $20 \%(P<0.05)$ and $\mathrm{MN}$-Colitis (0.28 and $0.09 \mathrm{~cm}^{2}$ respectively) by $40 \%(P<0.05)$ compared with $\mathrm{WN}$-Healthy $\left(0.35\right.$ and $0.17 \mathrm{~cm}^{2}$ respectively); there was no difference in total area. Cortical volume at mid-diaphysis was lower in WN-Colitis $\left(0.005 \mathrm{~cm}^{3}\right)$ by $30 \%(P<0.05)$ and MN-Colitis $\left(0.005 \mathrm{~cm}^{3}\right)$ by $30 \%(P<0.05)$ compared with WN-Healthy $\left(0.007 \mathrm{~cm}^{3}\right)$; there was no difference in total or trabecular volume. BMC at mid-diaphysis (total, trabecular, and cortical) was lower by 22,15 , and $55 \%$ (respectively; $P<0.05)$ in both WN-Colitis $(4.7,0.5$, and $3.9 \mathrm{mg}$ respectively) and $\mathrm{MN}$-Colitis (4.7, 0.5 , and $4.3 \mathrm{mg}$ respectively) compared with WN-Healthy $(6.0,1.1$, and $4.7 \mathrm{mg}$ respectively). vBMD at mid-diaphysis (total, trabecular, and cortical) was not different among the three groups (see Supplementary Table $\mathbf{4}$ online). 

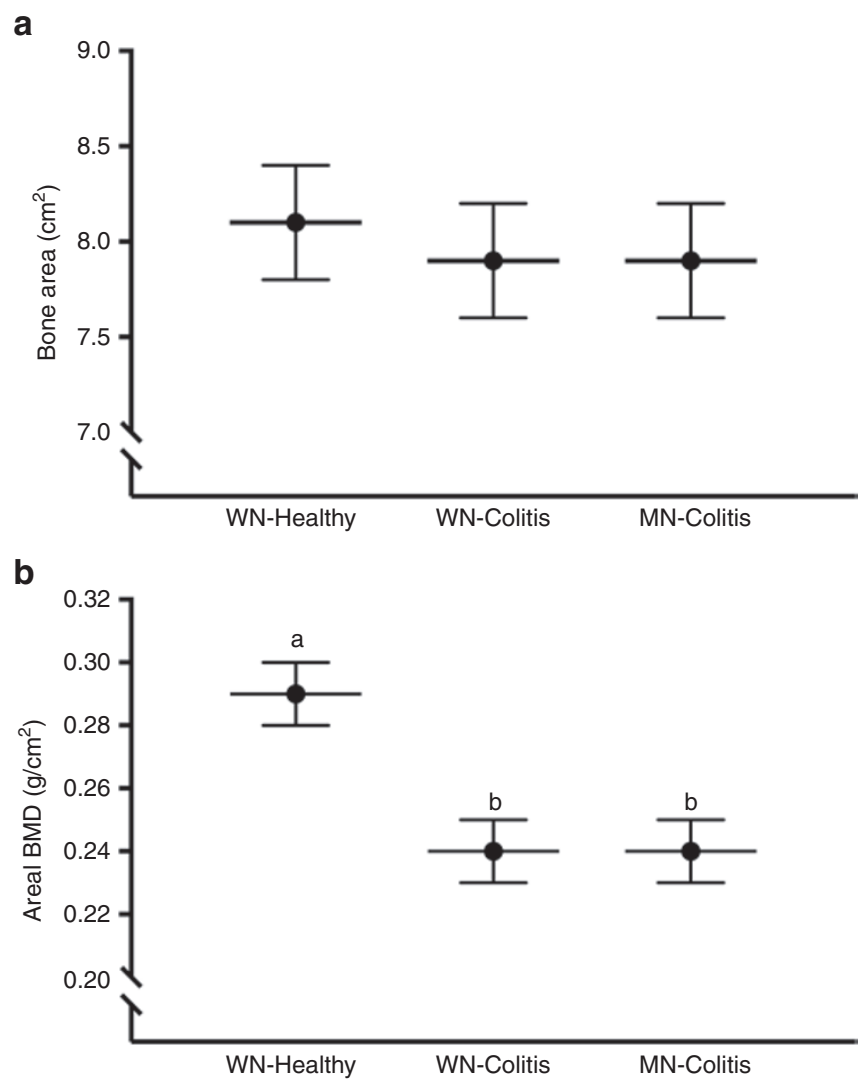

Figure 2. Dual-energy x-ray absorptiometry (DXA) analysis of femur whole bone area (a) and areal bone mineral density (b). Values are mean \pm pooled SE. Within each graph, means without a common letter differ, $P<0.05$. WN-Healthy, well-nourished healthy $(n=6)$; WN-Colitis, well-nourished colitis $(n=5)$; MN-Colitis, malnourished colitis $(n=5)$.

\section{Bone Strength}

Femur strength was uniquely affected by both colitis and nutritional status (Figure 5). Energy at break was lower in WN-Colitis $(956 \mathrm{~mJ})$ by $26 \%(P<0.05)$ and in MN-Colitis $(895 \mathrm{~mJ})$ by $31 \%(P<0.05)$ compared with WN-Healthy $(1295$ $\mathrm{mJ})$. Young's Modulus, stress, strain, work to failure, and modulus of toughness were not different among the three groups (see Supplementary Table S5 online).

\section{DISCUSSION}

This study presents a comprehensive analysis of bone in the growing piglet as a model for the growing child using precise imaging techniques. Using pQCT technology, we found lower vBMD (total, trabecular, and cortical) but similar area of femurs in piglets with colitis compared with healthy controls at all three regions analyzed (4, 8, and $12 \%$ from the growth plate). Analysis by $\mu \mathrm{CT}$ at mid-diaphysis found that trabecular and cortical area of femur were comprised by colitis, whilst vBMD remained the same. These data suggest that short-term colitis may compromise the mineralization of newly formed bone below the growth plate. Conversely, colitis may not alter density of bone at mid-diaphysis formed prior to administration of DSS, though further bone growth (i.e., bone area) seemed to be compromised. This notion may also be supported by the a
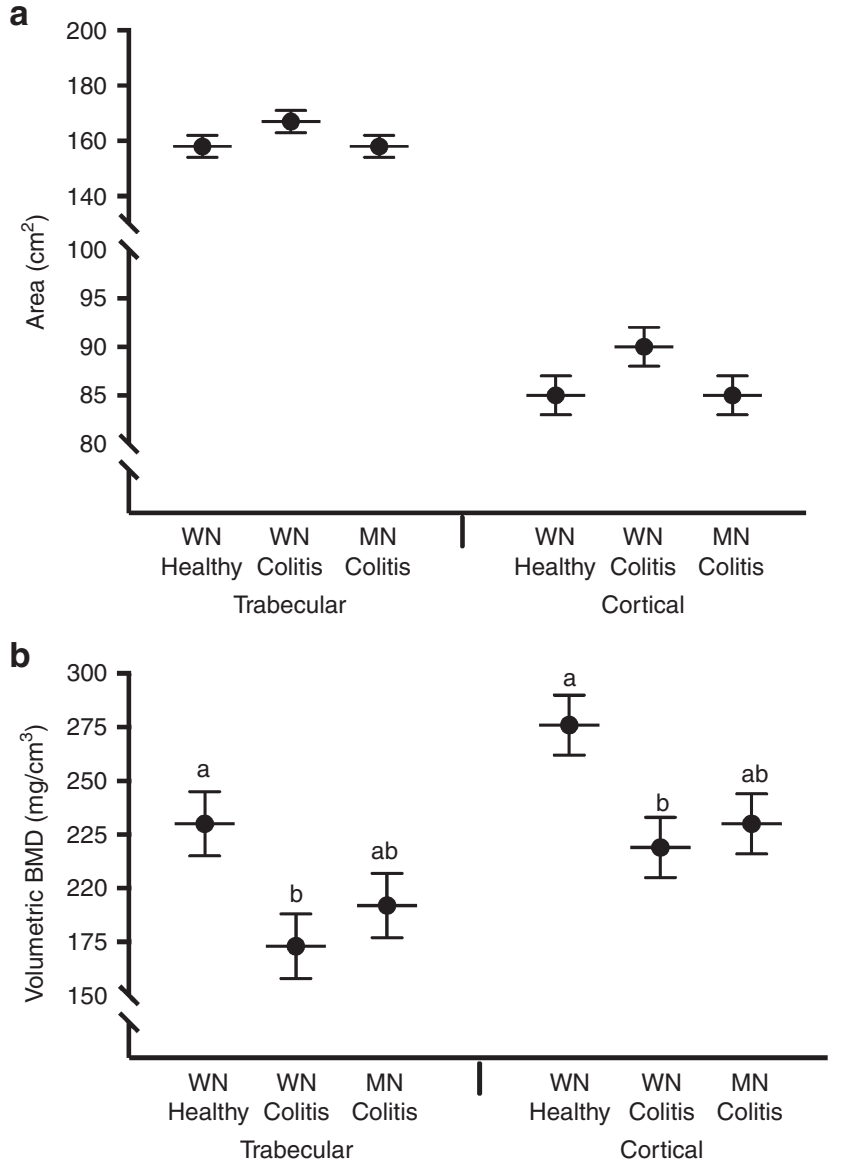

Figure 3. Peripheral quantitative computed tomography ( $\mathrm{QQCT}$ ) analysis of femur area (a) and volumetric bone mineral density (b) $4 \%$ from distal growth plate. Values are mean \pm pooled SE. Within each graph, means without a common letter differ, $P<0.05$. WN-Healthy, well-nourished healthy $(n=6)$; WN-Colitis, well-nourished colitis $(n=5)$; MN-Colitis, malnourished colitis $(n=5)$.

bone strength data. Femoral intrinsic strength was maintained (i.e., Young's Modulus and Modulus of Toughness), while the extrinsic property (i.e., energy at break) was lower in piglets with colitis. This suggests that piglets with colitis may be more sensitive to breaks when pressure is applied directly at mid-diaphysis where bone area is smaller (despite unaltered vBMD).

Our results are similar to previous work demonstrating 2 wk of DSS administration in 9-week old mice resulted in decreased trabecular bone volume and trabeculae number in distal metaphysis in mice with colitis compared with controls (19). Similarly, children newly diagnosed with Crohn's disease had significantly lower trabecular vBMD at tibial metaphysis compared with healthy controls, with significant improvement within the first 6 mo of treatment (25). However, it is unclear if the low vBMD in children is due to the inflammatory effects of colitis or altered nutrient metabolism that would limit mineralization of newer bone under the growth plate (26). In contrast to our work, others have suggested that children with IBD are not more likely to have increased fracture risk than healthy controls in the same age and gender category (26), while the 


\section{Articles | Vassilyadi et al.}

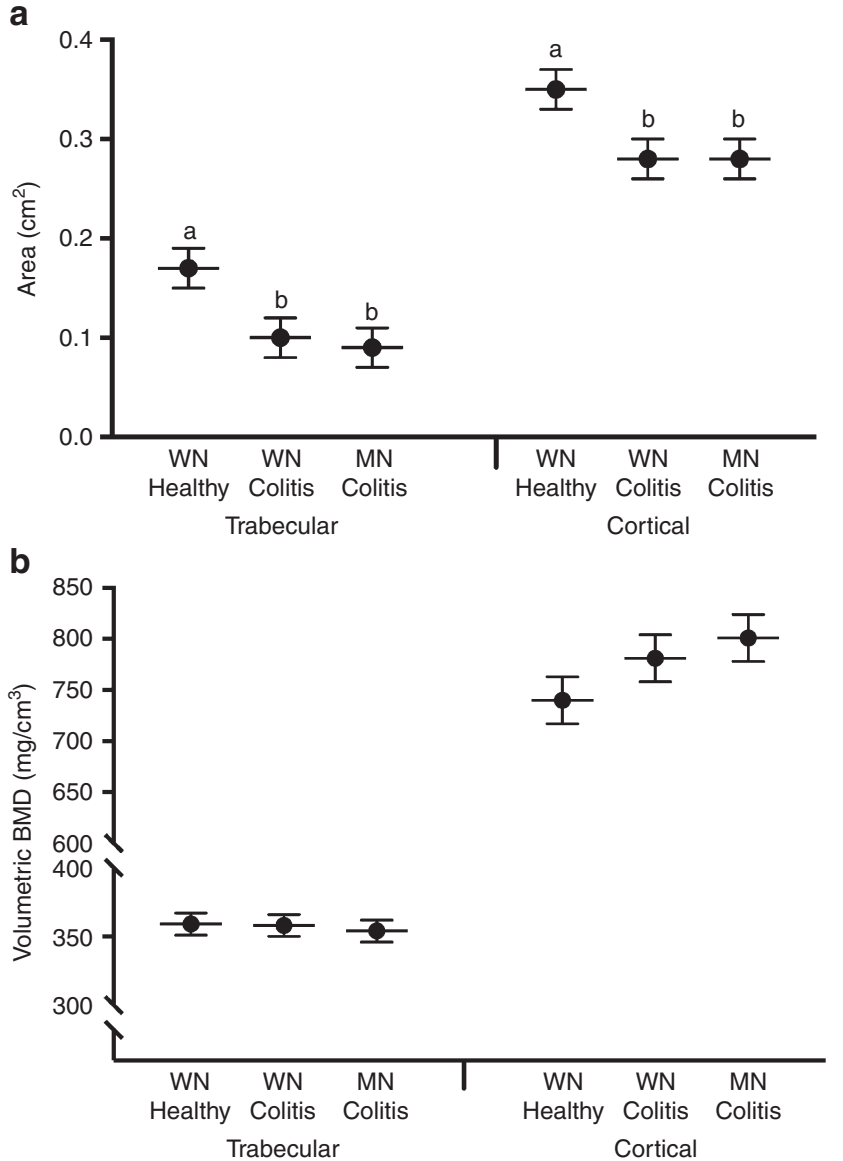

Figure 4. Micro computed tomography $(\mu \mathrm{CT})$ analysis of femur area (a) and volumetric bone mineral density (b) at mid-diaphysis. Values are mean \pm pooled SE. Within each graph, means without a common letter differ, $P<0.05$. WN-Healthy, well-nourished healthy $(n=6)$; WN-Colitis, well-nourished colitis $(n=5)$; MN-Colitis, malnourished colitis $(n=5)$.

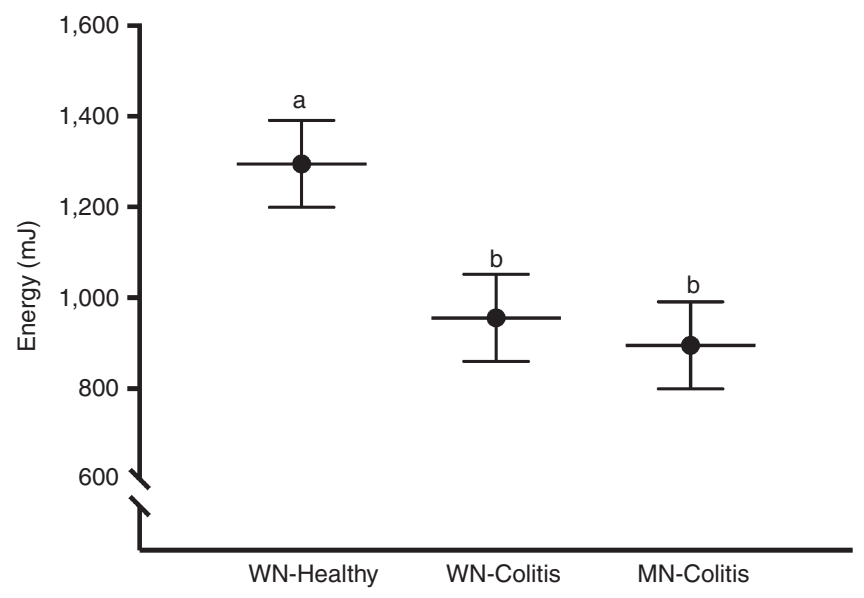

Figure 5. Energy at break of femur at mid-diaphysis. Values are mean \pm pooled SE. Within each graph, means without a common letter differ, $P<0.05$. WN-Healthy, well-nourished healthy $(n=6)$; WN-Colitis, wellnourished colitis $(n=5)$; MN-Colitis, malnourished colitis $(n=5)$.

prevalence of long bone fractures, as reported by parents in a survey, was also not different between children with IBD and their healthy siblings (27).
In our study, piglets were maintained on liquid formula diets delivered by intragastric feeding tube. Exclusive enteral nutrition has recently been used as the therapy of choice to induce remission in patients with Crohn's disease with $\sim 80 \%$ of patients entering remission after $8 \mathrm{wk}$ (28). Enteral feeds and steroids have similar efficacy in promoting remission (29) and patients receiving nutritional therapy showed less reduction in BMD than those receiving corticosteroids (30). Recently, after $8 \mathrm{wk}$ of exclusive enteral nutrition, markers of bone resorption and bone formation normalized to control levels, suggesting that adequate nutrition or nutritional therapy may normalize bone turnover by reducing inflammation (31).

We demonstrated that the superimposition of moderate protein energy malnutrition in piglets with short-term colitis did not compromise geometry of long bones. We cannot be certain if malnutrition had an effect, since all piglets were constantly fed an enteral formula consisting of high quality proteins with calcium, high vitamin $\mathrm{D}$, and adequate micronutrients. Therefore, our model of moderate protein energy malnutrition may not completely parallel to the human scenario. It is interesting to note that despite constant enteral feeds, bone geometry was compromised in piglets with colitis compared with their healthy controls, suggesting that the inflammatory response itself may have a greater short-term effect on bone than nutrition.

An important contribution of this study was to establish the conditions for comprehensive imaging with multiple platforms (DXA, pQCT, and $\mu \mathrm{CT}$ ). We found that aBMD (as analyzed by DXA) was lower in piglets with colitis than healthy controls, but vBMD of the whole femur (as analyzed by $\mu \mathrm{CT}$ ) was not different. Conversely, in children with IBD, there was no change in aBMD of the femoral neck (as determined by DXA); however, volumetric interpretation of the DXA data demonstrated low vBMD (9) after calculating bone mineral apparent density from a formula developed by Kröger and colleagues (32). This rationale stems from the idea that calculating vBMD using DXA analysis alone may not always reflect true vBMD-the area being measured is two-dimensional and does not include the depth and thickness of the bone, which is necessary for determining true BMC since minerals are spread throughout the entire bone (33). Based on our analysis, we found that $\mathrm{pQCT}$ and $\mu \mathrm{CT}$ are more precise in analyzing BMD compared with DXA, providing a volumetric analysis instead of a two-dimensional areal analysis. Additionally, we found that both pQCT and $\mu \mathrm{CT}$ are similarly precise in their analyses of total, cortical, and trabecular area, BMC, and vBMD.

There are several limitations to this study that must be addressed. Firstly, investigation of osteoblast/osteoclast activity, IGF-I levels and the receptor activator of nuclear factor-kappaB ligand (RANKL) and osteoprotegerin (OPG) system would have greatly completed the bone structure and strength outcomes to give a better understanding of bone development and integrity. Second, the sample size was small, ranging from $n=6$ to $n=8$ piglets; however, statistically significant data was homogenous and normally distributed. Lastly, the mechanism of how DSS induces colitis is not well described in literature and may not exactly represent how ulcerative colitis actually affects bone development in humans. 
In conclusion, we were able to use complementary imaging techniques to analyze bone geometry in a growing piglet model of DSS-induced-colitis. We have shown that short-term colitis compromises bone structure and bone strength of piglet femurs, independent of protein and energy malnutrition. Using DXA, we found that colitis decreased whole bone aBMD. Analysis with pQCT determined that colitis compromised vBMD of newly formed cortical and trabecular bone near the growth plate. The use of $\mu \mathrm{CT}$ at mid-diaphysis found that structural integrity of bone was maintained during colitis but lower area resulted in lower energy to break. Future investigations of osteoblast/osteoclast activity and expression of inflammatory mediators, bone growth factors would greatly complement the outcomes of our study and give a better understanding of bone development and metabolism. Inflammation plays a key role in the pathogenesis of bone loss in pediatric IBD and warrants further investigation toward therapies that may prevent and reverse the loss of bone.

\section{METHODS}

\section{Animals and Study Protocol}

Piglets ( $n=16$; 5-7 d old; Yorkshire $\times$ Landrace) were randomized to three treatment groups for the 14-day study, as previously described by Harding and colleagues $(21,22)$. Catheters were implanted in femoral and jugular veins (for blood sampling) and stomach (for diet and DSS administration) on day 1, using isoflurane (MTC Pharmaceuticals, Ontario, Canada) anesthesia (20). Constant intragastric feedings began $2 \mathrm{~h}$ after surgery and infused daily over $16 \mathrm{~h}$, reaching full feeding of $300 \mathrm{ml} / \mathrm{kg}$ by day 3 and adjusted daily based on weight. On day 4 , two groups received DSS $1 \mathrm{~g} / \mathrm{kg} / \mathrm{d}$ to induce colitis and, after $5 \mathrm{~d}$ of DSS, the presence of occult blood by Hemoccult (Beckman Coulter, ON, Canada) confirmed colitis.

Liquid diets were prepared in our laboratory using an egg albumin and whey protein base (21) to achieve nutritionally adequate energy intakes as per National Research Council (NRC) recommendations (34). Well-nourished groups received $100 \%$ of macro and micronutrient requirements (WN-Healthy and $\mathrm{WN}$-Colitis) and the malnourished group (MN-Colitis) received 50\% of macronutrient requirements to achieve energy intakes of 925 and $461 \mathrm{~kJ} / \mathrm{kg} / \mathrm{d}$, respectively. However, the MN-Colitis group received $>100 \%$ of the micronutrient requirements. Of particular interest to this study, vitamin D was supplied at $120 \%$ of NRC recommendation in all three groups.

The right hind leg from each piglet was removed at necropsy. Femurs were excised, cleaned of soft tissue, wrapped in saline soaked gauze and stored at $-20^{\circ} \mathrm{C}$. All animal procedures and protocols were reviewed and approved by the McGill University Animal Care Committee in accordance with the Canadian Council on Animal Care guidelines (35).

\section{DXA Analysis}

Each bone was scanned ex-vivo using DXA (Discovery Series 4500A densitometer; Hologic, Bedford, MA) and analyzed using QDR 4500A small animal software (version 12.5). Quality control was performed using a spine phantom, yielding a coefficient of variation (CV\%) for BMC and aBMD of $<0.5 \%$. Before positioning the femur, the length and width (at midpoint) was measured with a Fisherbrand traceable digital caliper (Fisher Scientific, Ottawa, ON, Canada). Each bone was scanned supine in a water bath where the water was $1 \mathrm{~cm}$ above the most prominent part of the bone. Data collected included bone area $\left(\mathrm{BA} ; \mathrm{cm}^{2}\right),(\mathrm{BMC} ; \mathrm{g})$, and $\left(\mathrm{aBMD} ; \mathrm{g} / \mathrm{cm}^{2}\right)$. All bones were scanned once, with the exception of the first bone, which was scanned three times.

\section{pQCT Analysis}

Each femur was scanned ex-vivo using a Stratec XCT 2,000 scanning unit (Stratec, Pforzeim, Germany). A tube made of \#2 plastic with the following dimensions was used: $15.5 \mathrm{~cm} \times 8.5 \mathrm{~cm} \times 0.5 \mathrm{~mm}$. The standard phantom was scanned with and without the plastic tube to rule out x-ray interference. Femurs were scanned in the supine position. The reference line was set before each scan using a scout view and placing the line at the lowermost point of the top of the distal epiphyseal plate. Gantry diameter was set to $60 \mathrm{~mm}$ and voxel size was $0.2 \mathrm{~mm}$. Cross sectional slices ( $1 \mathrm{~mm}$ thick) were made at each of five different sites: $4,8,12,40$ and 50\% from the reference line (NBthe reference line is placed $10 \%$ the length of the bone; therefore, the $40 \%$ site represents mid-diaphysis). The scout view speed was set to $30 \mathrm{~mm} / \mathrm{s}$ and the CT speed to $10 \mathrm{~mm} / \mathrm{s}$. To measure trabecular bone, a threshold of $107 \mathrm{mg} / \mathrm{cm}^{3}$ was used with a trabecular area of $65 \%$. To measure cortical bone a threshold of $524 \mathrm{mg} / \mathrm{cm}^{3}$ was used. For the three trabecular sites $(4,8$, and $12 \%)$, area and volumetric BMD were calculated for total, trabecular, and cortical bone. For the two cortical sites (40 and 50\%) cortical area and cortical density were calculated. Area is expressed in $\mathrm{mm}^{2}$ and density in $\mathrm{mg} / \mathrm{cm}^{3}$.

\section{$\mu C T$ Analysis}

Each bone was scanned ex-vivo using a LaTheta LCT-200 X-ray CT scanning unit (Hitachi Aloka, Tokyo, Japan) and analyzed using software version 1.3 (Hitachi-Aloka). Each bone was placed in a Lucite holder which was $60 \mathrm{~mm}$ in diameter. Positioning was standardized in the supine position, with the distal end of the bone facing the opening of the unit (specimen position was determined as "face down and head front"). Scan conditions were set as follows: pixel size $=60 \mu \mathrm{m}$, slice thickness $=120 \mu \mathrm{m}$, number of slices $=450$, pitch $=180 \mu \mathrm{m}$, and rotation angle $=360$ degrees. Each bone was scanned once with the exception of the first bone scanned on each day, which was scanned three times. The mid-diaphysis slice was then analyzed to provide parameters that were then used for the bone's moment of inertia, namely $x, x^{\prime}, y$, and $y^{\prime}$ as per Ferretti and colleagues (36). Parameters measured where: cortical thickness, planar volume (total, cortical, trabecular), BMC (total, cortical, trabecular), and vBMD (total, cortical, trabecular). Area is expressed in $\mathrm{cm}^{2}$, volume in $\mathrm{cm}^{3}, \mathrm{BMC}$ in $\mathrm{mg}$, and volumetric vBMD in $\mathrm{mg} / \mathrm{cm}^{3}$. All bones were scanned once, with the exception of the first bone, which was scanned three times.

\section{Bone Strength}

Biomechanical strength of each bone was tested ex-vivo by the 3 -point flexure method using an Instron v5544 (Instron, Canton, MA). Fulcrum span-length was set at $22.68 \mathrm{~mm}$. Bones were balanced between the two fixed supports in the supine position so that the load descends in the anterior to posterior direction. Mid-diaphysis was determined by dividing the length of the bone by two. A test speed of $2 \mathrm{~mm} / \mathrm{min}$ was used and bones were loaded until failure. The time to break was on average $2.3 \mathrm{~min}$. Load extension curves (n vs. mm) were constructed for each bone using Bluehill software version 3.0 (Instron). Extrinsic parameters measured were: energy at break, ultimate force, ultimate displacement, and work to failure. Intrinsic parameters measured were: stress, strain, Young's modulus, and modulus of toughness.

\section{Statistical Analysis}

All data was analyzed as one-way unbalanced ANOVA using the proc glm procedure of SAS Version 9.3. Least square means were calculated with pooled SE. Levene's test confirmed homogeneity of variance and the Anderson-Darling test confirmed normality. Tukey-Kramer test was used to determine statistical significance. A $P$-value of $<0.05$ was considered statistically significant. Data that were not homogenous or normally distributed were power transformed.

\section{SUPPLEMENTARY MATERIAL}

Supplementary material is linked to the online version of the paper at http://www.nature.com/pr

\section{ACKNOWLEDGMENTS}

We thank Paula Lavery for her invaluable assistance in training and calibration of the DXA, $\mu C T$, and INSTRON v5544.

\section{STATEMENT OF FINANCIAL SUPPORT}

This study was supported by Natural Sciences and Engineering Research Council of Canada (L.J.W. 183710-09 and 380302-09), McGill Graduate Excellence Fellowship (P.V.), and Canadian Foundation for Innovation (H.A.W. 217519, L.J.W. 23910). 


\section{Articles | Vassilyadi et al.}

Disclosure: There are no financial ties to products or potential/perceived conflicts of interests.

\section{AUTHOR CONTRIBUTIONS}

P.V. designed and carried out the research, analyzed the data, performed the statistical analysis and wrote the first complete draft of the manuscript; S.V.H. designed the original piglet research, carried out the piglet study and wrote the manuscript; T.J.H. designed and carried out the PQCT protocol and helped write the manuscript; H.A.W. designed the research and wrote the manuscript; L.J.W. conceived the study, carried out the piglet study, wrote the manuscript and had primary responsibility for the final content. All authors read and approved the final manuscript.

\section{REFERENCES}

1. Harris L, Senagore P, Young VB, McCabe LR. Inflammatory bowel disease causes reversible suppression of osteoblast and chondrocyte function in mice. Am J Physiol Gastrointest Liver Physiol 2009;296:G1020-9.

2. Semeao EJ, Stallings VA, Peck SN, Piccoli DA. Vertebral compression fractures in pediatric patients with Crohn's disease. Gastroenterology 1997;112:1710-3.

3. Thearle M, Horlick M, Bilezikian JP, et al. Osteoporosis: an unusual presentation of childhood Crohn's disease. J Clin Endocrinol Metab 2000;85:2122-6.

4. Stephens M, Batres LA, Ng D, Baldassano R. Growth failure in the child with inflammatory bowel disease. Semin Gastrointest Dis 2001;12: $253-62$.

5. Malik S, Mason A, Bakhshi A, et al. Growth in children receiving contemporary disease specific therapy for Crohn's disease. Arch Dis Child 2012;97:698-703.

6. El-Hodhod MA, Hamdy AM, Abbas AA, Moftah SG, Ramadan AA. Fibroblast growth factor 23 contributes to diminished bone mineral density in childhood inflammatory bowel disease. BMC Gastroenterol 2012;12:44.

7. Sylvester FA. Do bones crack under the effects of inflammatory bowel disease in children? J Pediatr Gastroenterol Nutr 2006;43:563-5.

8. van Hogezand R, Hamdy N. Skeletal morbidity in inflammatory bowel disease. Scand J Gastroenterol 2006;41: Suppl 243: 59-64.

9. Paganelli M, Albanese C, Borrelli O, et al. Inflammation is the main determinant of low bone mineral density in pediatric inflammatory bowel disease. Inflamm Bowel Dis 2007;13:416-23.

10. Lucarelli S, Borrelli O, Paganelli M, et al. Vertebral fractures and increased sensitivity to corticosteroids in a child with ulcerative colitis: successful use of pamidronate. J Pediatr Gastroenterol Nutr 2006;43:533-5.

11. Sawczenko A, Sandhu BK. Presenting features of inflammatory bowel disease in Great Britain and Ireland. Arch Dis Child 2003;88:995-1000.

12. Ghishan FK, Kiela PR. Advances in the understanding of mineral and bone metabolism in inflammatory bowel diseases. Am J Physiol Gastrointest Liver Physiol 2011;300:G191-201.

13. Jahoor F, Badaloo A, Reid M, Forrester T. Protein metabolism in severe childhood malnutrition. Ann Trop Paediatr 2008;28:87-101.

14. McCance RA. Overnutrition and undernutrition. II. Effects. Lancet 1953;265:739-45

15. Jones PR, Dean RF. The effects of Kwashiorkor on the development of the bones of the knee. J Pediatr 1959;54:176-84.

16. Ammann P, Bourrin S, Bonjour JP, Meyer JM, Rizzoli R. Protein undernutrition-induced bone loss is associated with decreased IGF-I levels and estrogen deficiency. J Bone Miner Res 2000;15:683-90.

17. Bozzini C, Bozzini C, Alippi R. Biomechanical properties of femoral shafts and bone tissue in protein-malnourished rats from weaning to adulthood. Comp Clin Path 2012;21:1159-1165.
18. Benight N, Burrin D, Barbara S. Intestinal metabolism of sulfur amino acids. In: Masella R, Mazza G, eds. Glutathione and Sulfur Amino Acids in Human Health and Disease. NJ: Wiley, 2009, pp 47-72.

19. Hamdani G, Gabet Y, Rachmilewitz D, Karmeli F, Bab I, Dresner-Pollak R. Dextran sodium sulfate-induced colitis causes rapid bone loss in mice. Bone 2008;43:945-50.

20. Wykes LJ, Ball RO, Pencharz PB. Development and validation of a total parenteral nutrition model in the neonatal piglet. J Nutr 1993;123: 1248-59.

21. Harding SV, Fraser KG, Wykes LJ. Probiotics stimulate liver and plasma protein synthesis in piglets with dextran sulfate-induced colitis and macronutrient restriction. J Nutr 2008;138:2129-35.

22. Harding SV, Adegoke OA, Fraser KG, et al. Maintaining adequate nutrition, not probiotic administration, prevents growth stunting and maintains skeletal muscle protein synthesis rates in a piglet model of colitis. Pediatr Res 2010;67:268-73.

23. Mackenzie ML, Warren MR, Wykes LJ. Colitis increases albumin synthesis at the expense of muscle protein synthesis in macronutrient-restricted piglets. J Nutr 2003;133:1875-81.

24. Miller ER, Ullrey DE. The pig as a model for human nutrition. Annu Rev Nutr 1987;7:361-82.

25. Dubner SE, Shults J, Baldassano RN, et al. Longitudinal assessment of bone density and structure in an incident cohort of children with Crohn's disease. Gastroenterol 2009;136:123-30.

26. Kappelman MD, Galanko JA, Porter CQ, Sandler RS. Risk of diagnosed fractures in children with inflammatory bowel diseases. Inflamm Bowel Dis 2011;17:1125-30.

27. Persad R, Jaffer I, Issenman RM. The prevalence of long bone fractures in pediatric inflammatory bowel disease. J Pediatr Gastroenterol Nutr 2006;43:597-602.

28. Day AS, Whitten KE, Lemberg DA, et al. Exclusive enteral feeding as primary therapy for Crohn's disease in Australian children and adolescents: a feasible and effective approach. J Gastroenterol Hepatol 2006;21:1609-14.

29. Heuschkel RB, Menache CC, Megerian JT, Baird AE. Enteral nutrition and corticosteroids in the treatment of acute Crohn's disease in children. J Pediatr Gastroenterol Nutr 2000;31:8-15.

30. Dear KL, Compston JE, Hunter JO. Treatments for Crohn's disease that minimise steroid doses are associated with a reduced risk of osteoporosis. Clin Nutr 2001;20:541-6.

31. Whitten KE, Leach ST, Bohane TD, Woodhead HJ, Day AS. Effect of exclusive enteral nutrition on bone turnover in children with Crohn's disease. J Gastroenterol 2010;45:399-405.

32. Kröger H, Vainio P, Nieminen J, Kotaniemi A. Comparison of different models for interpreting bone mineral density measurements using DXA and MRI technology. Bone 1995;17:157-9.

33. Leonard MB, Shults J, Elliott DM, Stallings VA, Zemel BS. Interpretation of whole body dual energy X-ray absorptiometry measures in children: comparison with peripheral quantitative computed tomography. Bone 2004;34:1044-52.

34. NRC. Nutrient Requirements of Swine. 10th edn. Washington DC: National Academy Press, 1998, pp 1-210.

35. Canadian Council on Animal Care. Guide to the Care and Use of Experimental Animals. Ottawa, ON: Bradda Printing Services, 1993, volume 1, pp 1-211, volume 2, pp 1-208.

36. Ferretti J, Capozza R, Mondelo N, Montuori E, Zanchetta JR. Determination of femur structural properties by geometric and material variables as a function of body weight in rats. Evidence of a sexual dimorphism. Bone 1993;14:263-270. 